University of Rhode Island

DigitalCommons@URI

Mechanical, Industrial \& Systems Engineering

Faculty Publications

$9-13-2020$

\title{
Identification and Modeling of a Variable Amplitude Fatigue Experiment Apparatus with Damaged Beam Specimen
}

Hewenxuan Li

David Chelidze

University of Rhode Island, chelidze@uri.edu

Follow this and additional works at: https://digitalcommons.uri.edu/mcise_facpubs

The University of Rhode Island Faculty have made this article openly available.

Please let us know how Open Access to this research benefits you.

This is a pre-publication author manuscript of the final, published article.

Terms of Use

This article is made available under the terms and conditions applicable towards Open Access

Policy Articles, as set forth in our Terms of Use.

\section{Citation/Publisher Attribution}

Li H., Chelidze D. (2021) Identification and Modeling of a Variable Amplitude Fatigue Experiment Apparatus with Damaged Beam Specimen. In: Kerschen G., Brake M.R., Renson L. (eds) Nonlinear Structures \& Systems, Volume 1. Conference Proceedings of the Society for Experimental Mechanics Series. Springer, Cham. https://doi.org/10.1007/978-3-030-47626-7_38

This Article is brought to you for free and open access by the Mechanical, Industrial \& Systems Engineering at DigitalCommons@URI. It has been accepted for inclusion in Mechanical, Industrial \& Systems Engineering Faculty Publications by an authorized administrator of DigitalCommons@URI. For more information, please contact digitalcommons-group@uri.edu. 


\title{
Identification and Modeling of a Variable Amplitude Fatigue Experiment Apparatus with Damaged Beam Specimen
}

\author{
Hewenxuan Li and David Chelidze \\ Nonlinear Dynamics Laboratory, \\ Department of Mechanical, Industrial and Systems Engineering, \\ University of Rhode Island, Kingston, RI 02881, USA
}

\begin{abstract}
The useful remaining life of engineering structures under variable amplitude (VA) fatigue loading remains a major unresolved engineering problem. The existing proposed life prediction models are usually based on empirical approximation from experimental results [? ? ]. The variable fatigue experiment apparatus in this extended abstract was designed for simulating structural fatigue with a high testing frequency, variable R-ratio as well as modifiable experimental layout [? ]. In previous studies, the inherent nonlinearity of the testing rig was detected, the obtained parameters allow one to properly use this testing rig within its linear region. As damage accumulates, however, the corresponding dynamic characteristics of the specimen alter accordingly. Therefore, proper modeling considering the interaction between the inherent nonlinearity and the damage induced nonlinearity for both 1) opening crack and 2) breathing crack is necessary for future fatigue life estimation under complex fatigue loading. Here, nonlinear system identification of the lately modified variable amplitude fatigue experiment apparatus is presented based on a combination of first-principles and data-driven modeling techniques. Eventually, structure-damage interaction dynamics will be described to model the underlying fatigue evolution and structural dynamics interactions.
\end{abstract}

Keywords: Nonlinear system identification, Modal parameter estimation, Damage identification, System-damage interaction, Hysteretic stiffness, Stick-slip dynamics

\section{CALIBRATION OF ACCELEROMETERS}

Before system identification, calibration of transducers was performed in order to make the resulting measurements comparable in their corresponding units [? ]. In the dynamic characterization, the main sensors being used are piezoelectric accelerometers and Eddy current displacement sensor. Two different series of accelerometers are being used, namely, PCB 333B42 and PCB U352C66 ICP accelerometers. Each series has three accelerometers, among which the measurements should be comparable such that their dynamic responses to the input are similar.

The six accelerometers are densely glued to one of the beam support to ensure no bias was introduced by the vibration mode of the beam support, see Fig. 1. The calibration was conducted under an input of sinusoidal signal with a frequency of $23 \mathrm{~Hz}$ and a magnitude of $200 \mathrm{mVpp}$. And the signals were amplified with a gain of 1 through PCB

\begin{tabular}{lllllll}
\hline DAQ Channel No. & 0 & 1 & 2 & 3 & 4 & 5 \\
\hline Sensor Chennel No. & 2 & 1 & 3 & 4 & 5 & 7 \\
\hline Sensor Series & & $333 \mathrm{~B} 42$ & \multicolumn{5}{c}{ U352C66 } \\
\hline Sensor No. & 12002 & 18444 & 18442 & 14469 & 20222 & 20224 \\
\hline Calibration Ratio (RMS) & 1.000 & 1.110 & 1.136 & 0.215 & 0.189 & 0.189 \\
\hline
\end{tabular}

Table 1: Table of relative calibration information. 

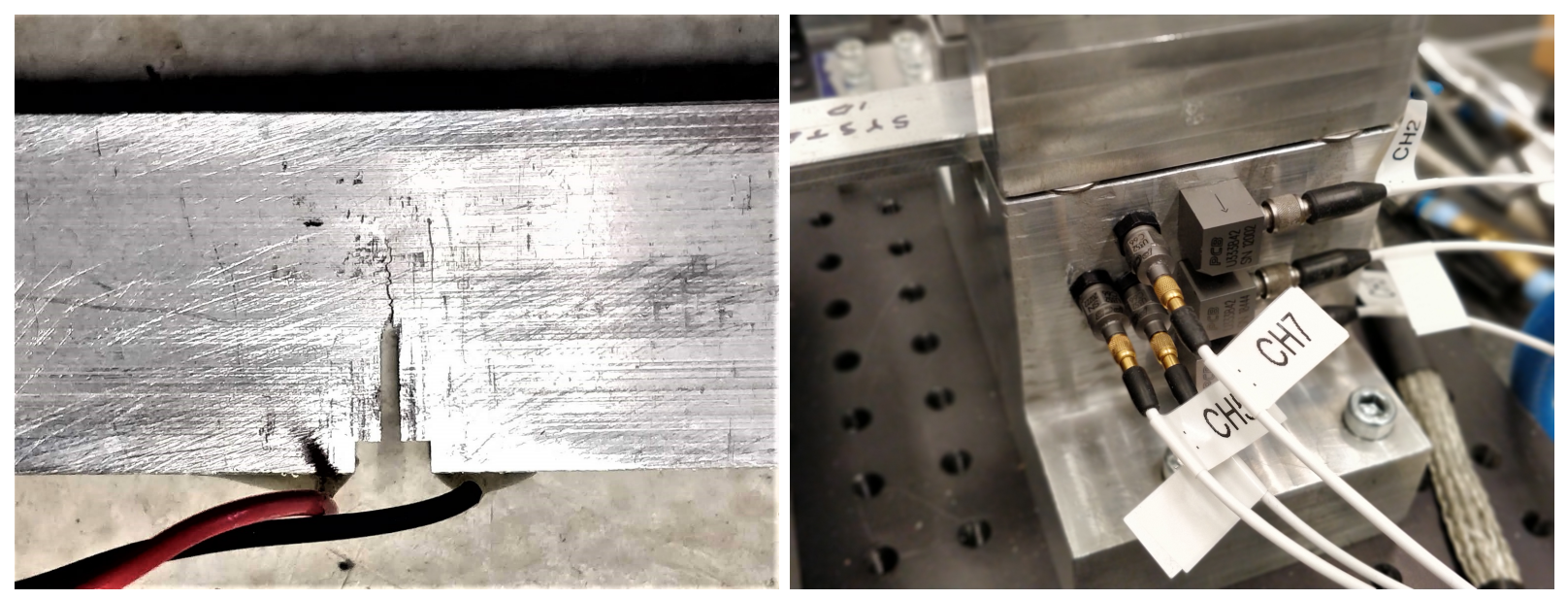

Fig. 1. Location of the accelerometers used during the calibration.

signal conditioner 498A03 and were not filtered. For the data acquisition, the out put signals were recorded by 6 channels on a National Instrument data acquisition card, with channel names a0 - a5 (CH1, ..., CH5, and CH7 from Fig. 1). All channels were sampled with a sampling frequency of $1000 \mathrm{~Hz}$. As for the frequency component of the acquired signals, a Welch's power spectral density estimation (periodogram) was obtained by adding a Hanning window (1024 samples long) with 50 percent overlapping between windowed signal segments. The raw and the RMS normalized load time history data in noise floor estimation were presented in Fig. 2. The noise floor estimation can be observed from Fig. 3. Since the fatigue experiments will be executed under $100 \mathrm{~Hz}$, the noise estimate is around $-24 \mathrm{~dB}$. The comparison between the two series of accelerometers indicates that the 333B46 series has batter signal to noise ratio (SNR) under $200 \mathrm{mVpp}, 23 \mathrm{~Hz}$ sinusoidal excitation, comparing to its counterparts. Aside from the $23 \mathrm{~Hz}$ resonance frequency, line noise related peaks can be found at $60 \mathrm{~Hz}, 120 \mathrm{~Hz}$ in the $333 \mathrm{~B} 42$ spectral density plot. Further investigation should be done in investigating the source of the $300 \mathrm{~Hz}$ peak, but for the sake of future experiments, this can be mitigated by the application of an antialiasing filter.

\section{DETECTION OF NONLINEARITY}

\section{Frequency Response Function Overlay}

Frequency response function is widely used in experimental modal analysis methodology which can also be utilized to detect nonlienarity of the system under investigation. Frequency response function overlay (FRFO) was used here to detect mainly the nonlinear stiffness of the system under investigation.

After calibrating the accelerometers, the system identification was established using sine swept excitation with up and down sweeping in order to detect nonlinearity in the system, if there is any. Frequency response functions can be obtained with various excitation level, this helps identify the certain kind of nonlinearity in the system caused by nonlinear stiffness. Therefore, three sets of swept-sine loading experiments were designed at three excitation amplitude (they are $100 \mathrm{mVpp}, 300 \mathrm{mVpp}$, and $500 \mathrm{mVpp}$, respectively). The preliminary FRFO results are illustrated in Fig. 4 from which it indicates that there exists softening spring nonlinearity since the resonance frequency depends on the forcing magnitude in a negatively correlated way. In another word, the resonance frequency of the system decreases with increasing forcing magnitude. Although the experiments were designed to detect the nonlinearity by up-sweeping and down-sweeping in frequency of sinusoidal loading, the results indicate relatively high damping in the system. Even though the acquired FRFs are influenced by damping, the results still support the observation of softening spring nonlinearity. The existence of a higher peak at a lower amplitude of excitation can be explained from the right sub-figure in Fig. 4 which shows the FRF in the vertical direction of the beam specimen. The higher peak is induced by the vertical resonance frequency at $80 \mathrm{~Hz}$. Therefore, the magnitude of the FRFO increases with increasing input amplitude.

\section{Nonlinear stiffness identification under static loading}

In previous laboratory location, nonlinear stiffness was detected and simplified to a cubic nonlinear stiffness. However, hysteresis behavior was observed and was not included in the modeling. More importantly, as damage accumulates, 

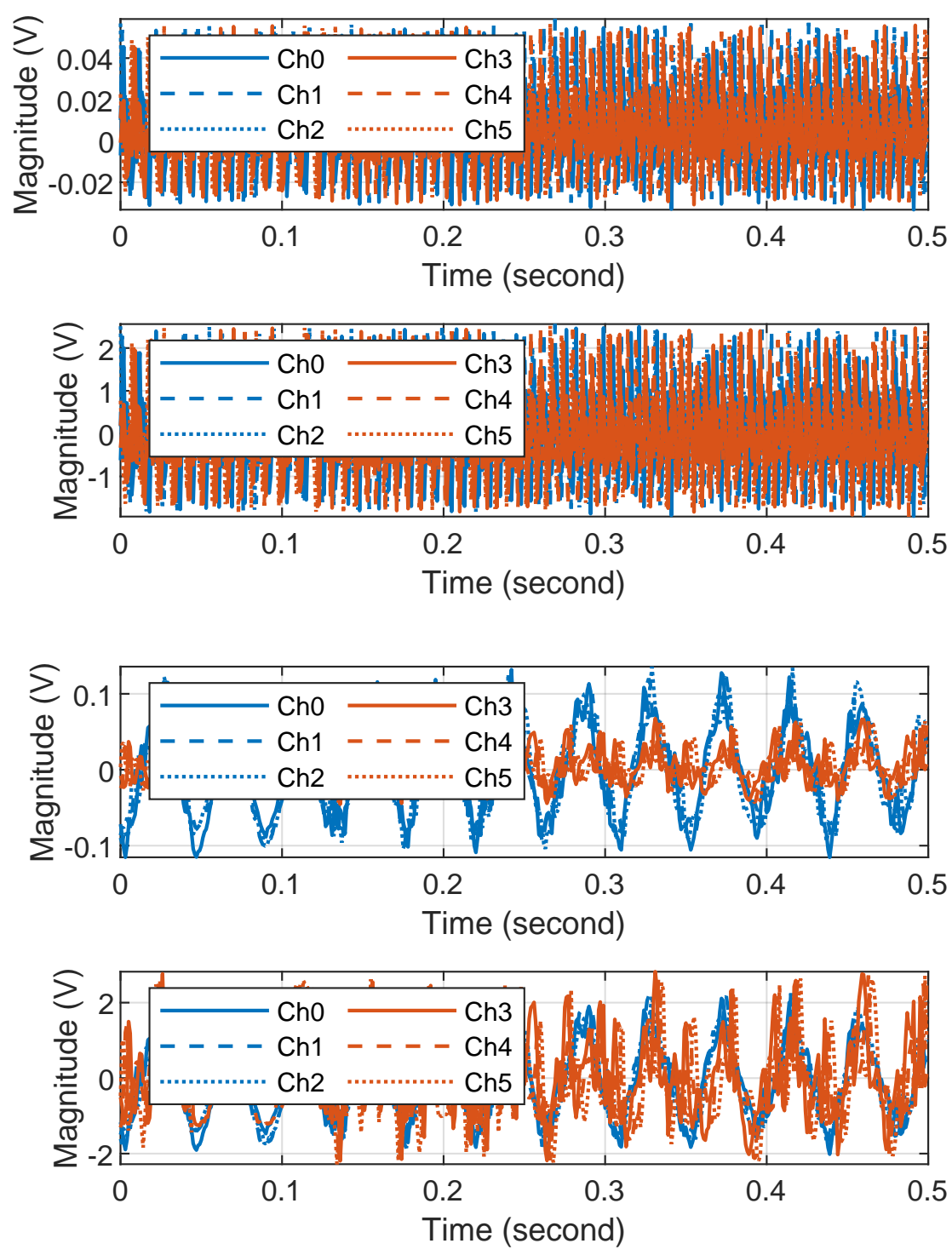

Fig. 2. Plot of load time history of the acquired signals without filtering. Upper two: raw and normalized load time history during noise floor estimation; lower two: raw and normalized load time history when system under a $23 \mathrm{~Hz}$, $200 \mathrm{mVpp}$ sinusoidal excitation.
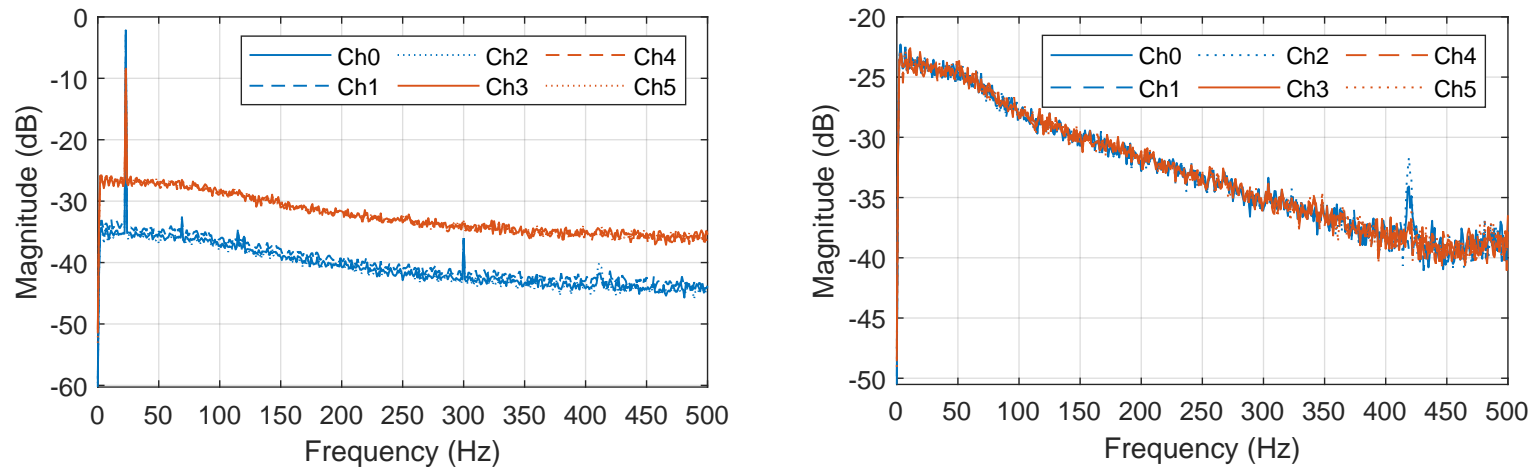

Fig. 3. Welch's periodogram of the acquired data for noise floor estimation of accelerometers. 

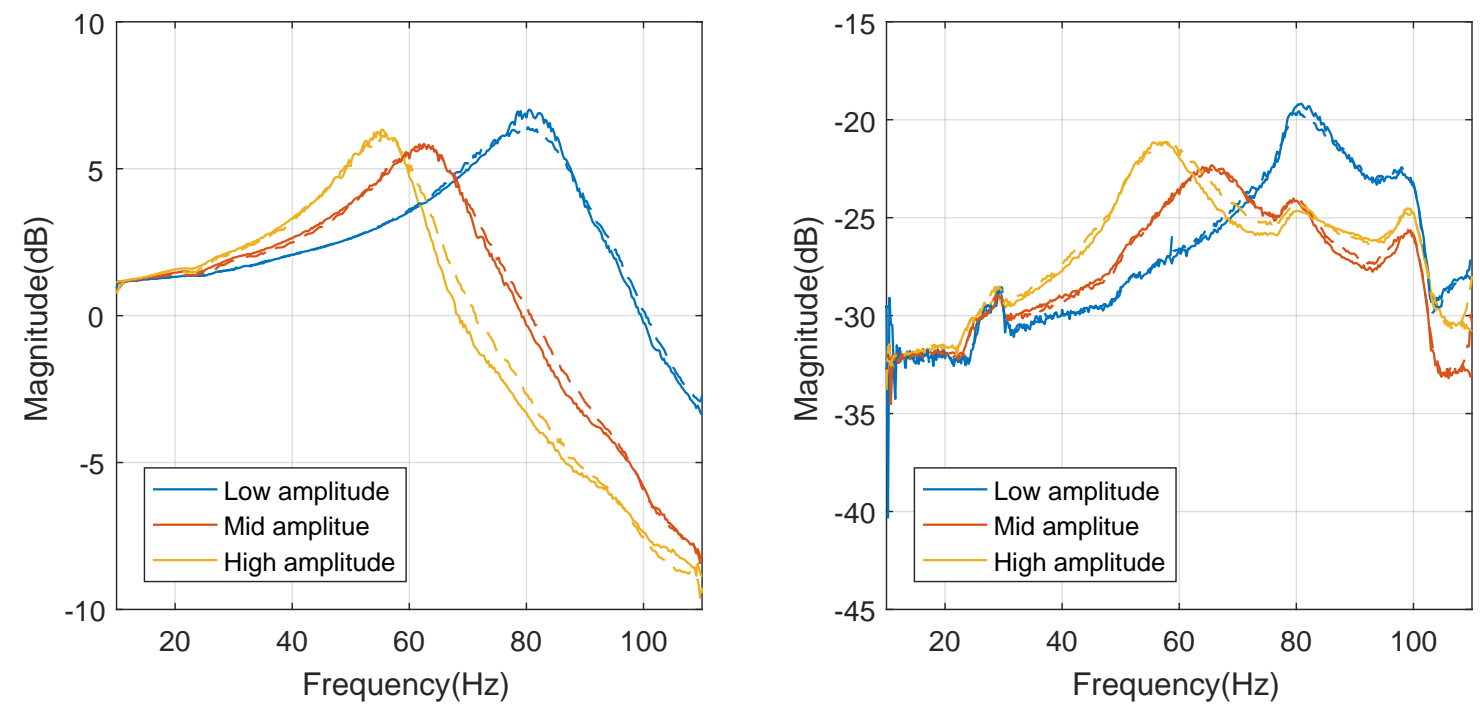

Fig. 4. Frequency response function overlay results indicate softening spring nonlinearity in the system under investigation.
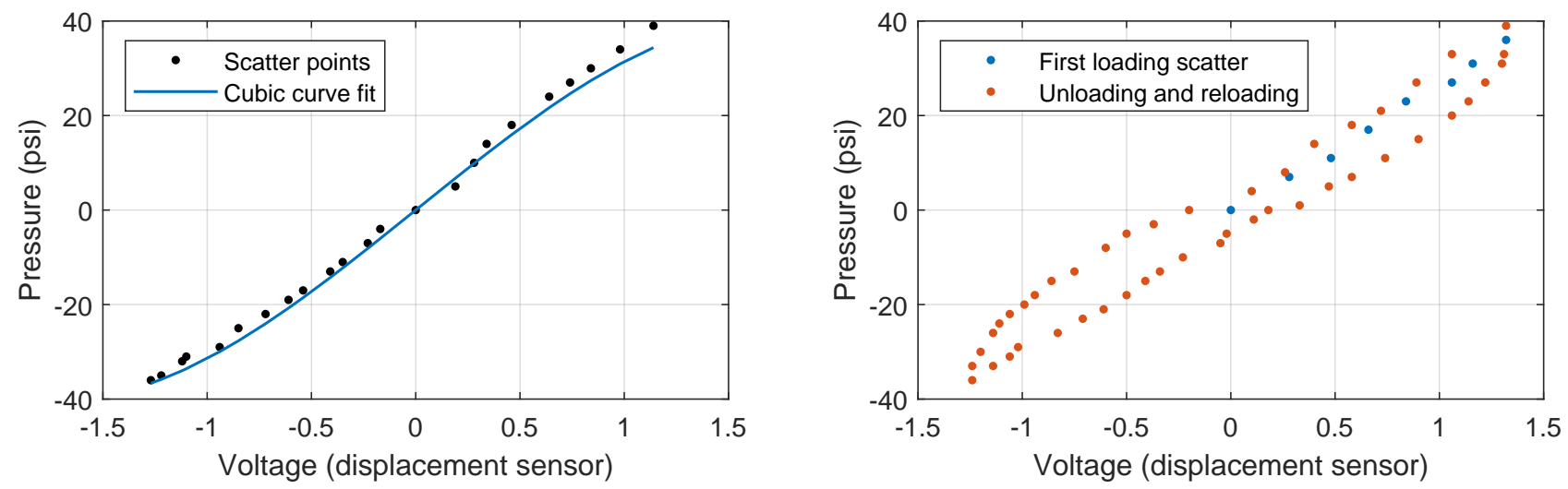

Fig. 5. Left: raw data of the deflection-pressure. 
the stiffness characteristics can be much more complex, hence, a more judicious examination has to be considered. Here, aside from the cubic softening nonlinear stiffness, the reason behind the hysteresis was investigated by loading and unloading the system statically through the pneumatic cylinders. The deflection-pressure curves were obtained by measuring the deflection using a Lion precision eddy current displacement sensor, and the corresponding pressure was measured by two SSI technology P51 series pressure sensors. Two sets of experiments were designed and executed. The pressure in the two cylinders were set to equal values before initiation of the experiments. A static load will then be applied to the beam from one side by increasing the pressure in the cylinder on the same side. The first experiment was a pure loading experiments during which the pressure setup will increase monotonically until 36psi while the second experiment was designed to explore the hysteresis behavior in the force-displacement relation. This result indicates softening spring nonlinear stiffness (which can be described by a cubic function) as well as hysteretic behavior, see the right sub-figure in Fig. 5. For now, it is hard to designate a good model for this behavior since dynamic behavior that relates force-velocity will reveal the hysteretic behavior in detail [? ? ].

\section{FUTURE WORK}

First, dynamic characterization of nonlinear stiffness will be conducted using restoring force reconstruction to further understand the dynamics of the system under investigation. Later on, damage identification with the comparison of a long notched beam and beams with equal length macro crack will be performed to provide empirical data for future parameter estimation and model updating. The last step is to establish a system model with time varying system parameters that takes the damage propagation into consideration. This will be further served to produce accurate results in real time fatigue life prediction modeling.

\section{ACKNOWLEDGEMENTS}

This work is supported by the National Science Foundation Grant No. 1561960. 